\section{Aplicaciones torácicas del ultrasonido}

\author{
SEBASTIÁN FERNÁNDEZ-BUSSY ${ }^{1}$, GONZALO LABARCA ${ }^{2}$, \\ MARIO LANZA ${ }^{3}$, ERIK FOLCH ${ }^{4}$, ADNAN MAJID $^{4}$
}

\section{A review on thoracic ultrasound}

The use of thoracic ultrasound as a diagnostic tool in the emergency department, intensive care unit or in patients with pulmonary diseases is increasing steadily. It is used to guide percutaneous tracheostomies, to assess pleural effusions, to rule out pneumothorax, and to guide the placement of endovascular and pleural catheters. It is also useful in the assessment of patients with dyspnea. The aim of this review is to provide the practical and technical basics for the use of this diagnostic tool among internists and specialists in pulmonary diseases.

(Rev Med Chile 2016; 144: 903-909)

Key words: Dyspnea; Pleural Diseases; Pleural Effusion; Pneumothorax, Ultrasonography.
'Sección de Neumologia Intervencional. Clinica Alemana de SantiagoUniversidad del Desarrollo. Santiago, Chile.

${ }^{2}$ Residente de Medicina Interna. Facultad de Medicina. Pontificia Universidad Católica de Chile. Santiago, Chile.

${ }^{3}$ División de Neumología Intervencional. Universidad Autónoma de Honduras. Tegucigalpa, Honduras. ${ }^{4}$ Division of Thoracic Surgery and Interventional Pulmonary. Beth Israel Deaconess Center-Harvard Medical School. Boston, Massachusetts, United States.

Los autores declaran no tener conflicto de interés.

Sin ayuda financiera.

Recibido el 1 de julio de 2015, aceptado el 12 de enero de 2016.

Correspondencia a:

Dr. Sebastián Fernández-Bussy

Dirección: Manquehue Norte 1410,

Santiago. Chile

Teléfono +56 222101428

sfernandezbussy@alemana.cl
E l empleo del ultrasonido se ha incrementado en la última década, en especial por el riesgo mínimo de su uso, el diagnóstico rápido, ausencia de riesgo de radiación, bajo costo, el ser portable $y$, definitivamente, por ayudar a tomar una decisión médica en forma rápida ${ }^{1}$. El impacto en el estudio de la cavidad torácica y, más recientemente, el desarrollo de sondas ultrasonográficas para el estudio de patología de la vía aérea, ha dado al neumólogo un impulso para obtener diagnósticos más claros y definitivos. Además, ha sido de utilidad en la guía de procedimientos invasivos, reduciendo las complicaciones ${ }^{2,3}$. Las indicaciones en el estudio de la patología torácica benigna y maligna son múltiples, como guía para el drenaje de líquido pleural, toma de biopsias dirigidas y guía para la colocación de catéteres torácicos ${ }^{4}$.

El objetivo de la presente revisión es dar a conocer aspectos técnicos, así como indicaciones de procedimientos en los cuales el uso del ultrasonido puede tener un rol clave tanto para el médico internista, como para el intensivista o el neumólogo.

\section{Aspectos técnicos}

El ultrasonido depende del principio piezoeléctrico, en el que impulsos eléctricos son dirigidos al transductor, el cual está compuesto por minúsculas piezas de cerámica que al estar en contacto con las ondas eléctricas generan movimientos que se traducen en ondas sonoras ${ }^{1}$. Estas últimas, al enfrentarse a un tejido, se comportarán de diferente forma, dependiendo del grado de impedancia que el tejido le imponga. Algunas ondas sonoras se propagan en los tejidos, en especial aquellos con alto contenido de agua, como el hígado, músculo, consolidaciones y tumores, mientras otras ondas no son propagadas y retornan al mismo transductor, lo que es llamado reflectancia. Este fenómeno es mayor en el tejido no comprimible, como el hueso, y observable también en la presencia de aire. La imagen procesada dependerá de las propiedades de las ondas sonoras, las que hay que destacar: 1) Longitud de onda, definida como la distancia entre dos bandas adyacentes. Esta capacidad de compresión y extensión de la onda de sonido permite definir características de 
los tejidos; 2) Frecuencia, definida por el número de ondas por segundo, con su unidad en Hertz; 3) Período, definido por el tiempo de la longitud de onda, y finalmente 4) Velocidad, definida por la frecuencia por la longitud de onda. Las propiedades acústicas de los tejidos producirán diferentes características ecogénicas, dando diferentes tonos de blanco-gris-negro. Las diferentes ecogenicidades de los diferentes tejidos son:

1. Hueso y aire: muy ecogénico/hiperecoico (refleja gran parte de las ondas)/tono blanco.

2. Músculo: ecogénico/hiperecoico.

3. Hígado y riñón: ecogénico/isoecoico, eco menos ecogénico.

4. Grasa: hipoecogénico/hipoecoico, tono obscuro.

5. Sangre y fluidos: hipoecogénico/hipoecoico, tono muy obscuros ${ }^{1,4}$.

Los transductores del ultrasonido permiten manejar diferentes frecuencias de ondas, los de alta frecuencia darán mejor definición en la imagen, pero con poca penetración hacia los tejidos, mientras que los transductores de baja frecuencia permiten mejor penetración en los tejidos, pero con menor resolución en la imagen. Para la valoración del tórax se ha recomendado la utilización de un transductor convexo de 5 a 7,5 MHz, lográndose excelente resolución de la pared del tórax y estructuras vasculares, útil en la canalización de la vena yugular interna y vena subclavia. El transductor de 3,5 a $5 \mathrm{MHz}$ es ideal para evaluar estructuras profundas del tórax, como el espacio pleural. El ultrasonido endo-bronquial radial emplea un transductor de $20 \mathrm{MHz}$, permitiendo mejor definición de la pared tráqueo-bronquial y de estructuras adyacentes. Mientras que el ultrasonido endo-bronquial lineal utiliza transductor de 7,5 Mhz, permitiendo la visualización de estructuras más profundas en el mediastino. Se puede observar el incremento acústico (hiperecoico) de un objeto de mínima atenuación (como el caso del aire en los alvéolos), o la sombra acústica en la que se observa una región anecoica tras un objeto de alta reflectancia (como en el caso de la pleura) ${ }^{1}$.

Se puede ajustar y mantener una imagen ultrasonográfica balanceada mediante el manipuleo de la ganancia de ecogenicidad, logrando mejor definición. También se puede ajustar la profundidad de la imagen para definir mejores detalles en los tejidos. Las estructuras que pueden

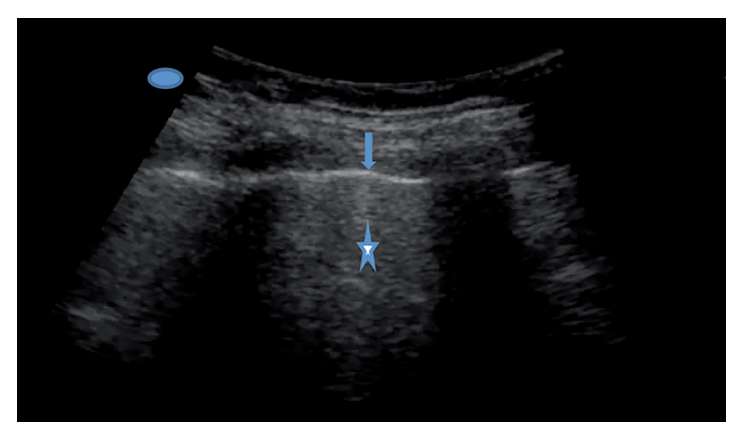

Figura 1. Ultrasonido normal, líneas horizontales hiperecogénicas (reverberación del pulmón) (asterisco), pleura (flecha).

ser observadas en el ultrasonido torácico son: tejidos blandos; pleura parietal y visceral, que en ausencia de patología pleural éstas suelen medir menos de $2 \mathrm{~mm}$ de grosor y se distinguen como dos líneas hiperecoicas; el espacio pleural, en ausencia de patología suele ser difícil de definir, ya que es prácticamente un espacio virtual menor de $0,3 \mathrm{~mm}$ de grosor; el tejido pulmonar, que se distingue por líneas hiperecoicas que representan el artefacto producido por el aire que entra y sale de los espacios alveolares ${ }^{3}$ (Figura 1).

El "signo del deslizamiento" está causado por el movimiento del tejido pulmonar subyacente a la pleura visceral; el diafragma, definido por ser una línea hiperecoica que sigue los movimientos respiratorios. Otros puntos de referencia útiles son: el parénquima hepático y renal en el lado derecho, y el riñón y bazo en el lado izquierdo ${ }^{4}$.

El transductor debe ser orientado en sentido cefálico y en forma perpendicular a la anatomía del tórax, de manera que la imagen que se observe sea real del sitio examinado y no de estructuras sobrepuestas. Aunque el ultrasonido de tórax tiene limitaciones al compararse con la tomografía axial computarizada de tórax para la evaluación del parénquima pulmonar y el mediastino, tiene ventajas en la caracterización del espacio pleural, alcanzando una mejor visualización, medición del volumen pleural, identificación de adherencias, etc. ${ }^{5}$.

\section{Ultrasonido en derrame pleural}

La presencia de líquido en el espacio pleural puede ser definida con facilidad. En el Modo-B, el derrame pleural puede ser definido por ser 
anecoico (muy obscuro), limitado por la imagen del diafragma, banda delgada hiperecoica que sigue los movimientos de la respiración, y por el pulmón colapsado, isoecoico. Esta zona donde se distribuye el líquido suele estar libre de ecos y es homogénea, sin gas en su interior y sin cambios en su ecogenicidad durante los movimientos de inspiración y expiración. Cuando el derrame es significativo y comprime el pulmón, este aparece como una imagen de consolidación triangular isoecoica flotando en el espacio pleural. En Modo-M, el derrame pleural puede ser sospechado por una banda anecoica que separa las bandas de los tejidos blandos con la línea hiperecoica que representa la pleura visceral, y por la forma sinusoidal que adquiere esta última. El ultrasonido permite distinguir si el líquido en la cavidad pleural es fluido o denso, esto último, indicando la presencia de detritos proteínicos (exudado) ${ }^{4}$. En caso de derrames pleurales complicados se pueden identificar líneas septales que dividen la cavidad en lóculos, estos septos pueden tener diferentes grosores y formar lóculos de diferentes dimensiones. Se puede medir el espesor de la pleura, identificar si existen engrosamientos, de carácter regular o irregular, siendo estos secundarios a pleuritis; además, se pueden observar placas pleurales, localización de masas y consolidaciones subpleurales, determinando en su interior sombras lentiformes hiperecoicas, lo que representa el equivalente al broncograma aéreo observado en otros métodos de imagen ${ }^{4}$. Se puede estimar la cantidad de líquido en la cavidad pleural, midiendo la separación entre la pleura costal y la pleura visceral, siendo más sensible que la radiografía de decúbito lateral. Se estima una cantidad de líquido pleural de $170 \mathrm{ml}$ si la separación es de $1 \mathrm{~cm}$, de $580 \mathrm{ml} \mathrm{si}$ la separación es de $2,5 \mathrm{~cm}$ y de $1.000 \mathrm{ml}$ si esta separación es de $4 \mathrm{~cm}^{5-7}$ (Figura 2a). Es posible identificar el derrame subpulmonar, así como la parálisis frénica, o elevación del hemidiafragma por afectación abdominal ${ }^{3,7}$.

\section{Ultrasonido para guiar toracocentesis}

El ultrasonido es un procedimiento seguro, rápido y eficaz, para definir el sitio específico de colocación de catéter o sonda para el drenaje de líquido pleural, pudiéndose identificar estructuras adyacentes como diafragma, parénquima pulmo- nar o vísceras abdominales. La toracocentesis no guiada por ultrasonido puede tener complicaciones graves como el neumotórax, reportado entre 20 y $39 \%$. Esta complicación puede ser reducida hasta $0 \%$ cuando el procedimiento es guiado por ultrasonografía ${ }^{8}$. Idealmente, debe ser realizado por el especialista en el mismo momento que la toracocentesis, ya que no se ha visto una reducción de la incidencia de neumotórax cuando la punción torácica es realizada por el radiólogo, pero en otro momento. Una situación especial es cuando el derrame pleural es mínimo, o cuando el paciente no puede ser colocado en una posición sentada, como los sometidos a ventilación mecánica; en estos casos, el rendimiento es significativamente mayor al compararse al método sin guía ultrasonográfica. El Doppler es de ayuda para identificar estructuras vasculares en casos de haber dificultad en identificar órganos abdominales ${ }^{4}$.

Idealmente, el paciente debe ser colocado en posición sentada, con la espalda erguida y los brazos cruzados hacia delante descansando sobre una mesa, con ello se logra que la escápula pueda estar fuera del campo de procedimiento, además de permitir la apertura de los espacios intercostales. Cuando el paciente no puede ser colocado en posición sentada, como pueden ser los pacientes en ventilación mecánica, se puede realizar en posición supina, lateralizado al lado contrario del sitio que será examinado, con la cabecera de la cama elevada, el brazo sobre el tórax y traccionado hacia el hombro contralateral. También puede realizarse con el brazo del paciente sobre su cabeza de manera que permita la separación de los arcos costales. El transductor debe ser colocado en la orientación cefálica y perpendicular a la pared torácica. Se debe recorrer los espacios intercostales identificando las estructuras internas como son el hígado, riñones, bazo, el diafragma, el espacio pleural y el parénquima pulmonar, definiendo así el mejor sitio, debiendo ser confirmado en varias visualizaciones (Figura 2b).

Se marca el sitio en forma indeleble, se ajusta la imagen con la perilla de ganancia y de profundidad definiendo la profundidad de la piel hasta el punto blanco del procedimiento y así tener noción sobre cuánto debe ser introducido el trocar junto con el catéter. Siempre debemos ingresar en el borde superior de la costilla, para evitar daño al paquete vásculo-nervioso. Cuando el derrame es significativo, debe evitarse el drenaje masivo de líquido, 

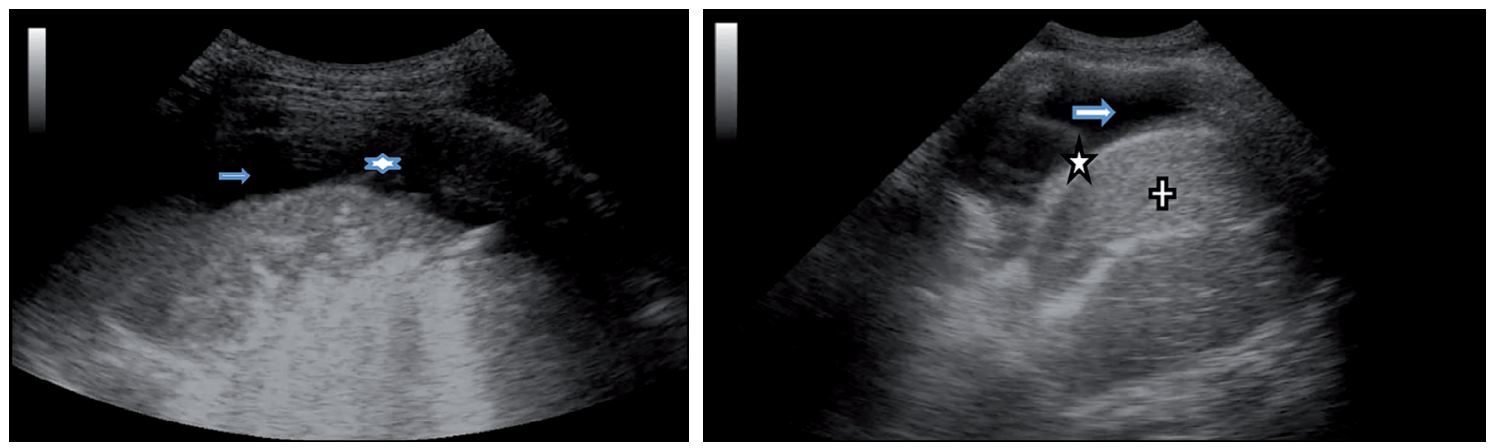

Figura 2. A: Derrame pleural (flecha), fibrina (asterisco); B: Derrame pleural (flecha), diafragma (asterisco), hígado (+).

por el riesgo de edema pulmonar por reexpansión; se recomienda no remover más de $1.500 \mathrm{ml}$ de líquido en cada sesión de toracocentesis. También es de utilidad la medición de la presión pleural y su seguimiento mientras se realiza la extracción de líquido, si se llega a una presión en la cavidad pleural de $-20 \mathrm{~cm}$ de agua, debe evitarse seguir drenando líquido, ya que el cambio significativo de presión es premonitorio de edema pulmonar por reexpansión ${ }^{2,9,10}$.

Se ha descrito la presencia de neumotórax luego del drenaje del líquido pleural guiada por ultrasonido aun sin haber habido lesión del parénquima pulmonar, ello puede ser debido al neumotórax ex-vacuo. Esto puede ser observado en la obstrucción bronquial de cualquier etiología, como lesión tumoral, cuerpo extraño, tapones de moco, mal posición de tubo endotraqueal, colapso segmentario o lobar, o atelectasia crónica. También puede ser debida a la presencia de engrosamiento pleural que impide la expansión pulmonar. En estos casos, la presencia de líquido pleural compensa la presión negativa incrementada en el espacio pleural, de manera que al drenar el líquido y haber imposibilidad del pulmón a expandir, se forma el neumotórax ex-vacuo. Con la acumulación seguida de líquido se forma un hidroneumotórax. La manometría pleural es útil en identificar el pulmón atrapado o inexpandible. La medición de la presión pleural es un procedimiento rápido, sencillo y que da información útil sobre la posibilidad de éxito en el intento de lograr la expansión pulmonar completa. Los cambios significativos en la presión al drenaje de poco líquido es indicativo de que el pulmón está atrapado ${ }^{9,11,12}$. En estos casos de "pulmón atrapado", los catéteres pleurales tunelizados para manejo paliativo son muy útiles ${ }^{13}$.

\section{Biopsias pleurales y transtorácicas}

Es clara la utilidad del ultrasonido en la toma de biopsias pleurales, identificando al punto más idóneo para la punción o para el ingreso del toracoscopio (en pleuroscopías), eligiendo el lugar con menos tabiques o más líquido en la cavidad pleural. Lesiones pulmonares en contacto con la pleura ofrecen una oportunidad para realizar biopsias transcutáneas guiadas por ultrasonido. Los tumores pulmonares se comportan como lesiones predominantemente hipoecogénicos ${ }^{3}$. La Sociedad Británica del Tórax ha incluido al ultrasonido en el algoritmo del diagnóstico del mesotelioma ${ }^{2,14}$.

La sensibilidad diagnóstica es mayor para lesiones malignas y tuberculosis. En el mesotelioma maligno tiene resultados similares a los obtenidos con la toracoscopia, con una precisión de $83 \%{ }^{3}$. Si bien la tomografía computarizada (TC) es la prueba de elección en la estadificación del mesotelioma, el ultrasonido nos permite corroborar la afectación de la pared torácica y pleura diafragmática, siendo su presentación habitual como nódulos de $5 \mathrm{~mm}$ en pleura parietal.

La ultrasonografía puede ayudar en el diagnóstico bacteriológico y drenaje de abscesos pulmonares que se implantan en la pleura, procedimiento similar a la valoración de masas pleurales. Se identifica la ausencia del deslizamiento pulmonar, efecto por inflamación local de la pleura. El Doppler puede visualizar posibles vasos aberrantes dentro del absceso ${ }^{13}$. 
La ultrasonografía es importante en la valoración y seguimiento de las neumonías y sus posibles complicaciones, como derrames paraneumónicos o abscesos intrapulmonares. La ultrasonografía es más sensible que la TC para evaluar invasión y compromiso de la pared torácica, siendo las neoplasias las que contribuyen a más destrucción de la morfología pulmonar normal y afectación de bronquios y vasos ${ }^{13}$.

\section{Edema pulmonar agudo}

El ultrasonido ha sido poco empleado para la identificación del edema pulmonar, sin embargo, se ha demostrado su utilidad en el diagnóstico temprano y rápido de esta condición. Para su identificación hay que estar familiarizado con algunos signos, como el signo de "la cola de cometa", que en el Modo B se observan como áreas hiperecoicas que emanan de la línea pleural (Figura 3a).

"Las líneas B" son artefactos producidos por el aire alveolar y representa un signo de normalidad del pulmón, sin embargo, cuando estas líneas son más acentuadas y difusas pueden representar engrosamiento del espacio intersticial o la presencia de líquido en el septo interlobar, pudiendo ser indicio de edema pulmonar ${ }^{4}$. Cuando se cambia a Modo M, el parénquima pulmonar normal se observa granular, simulando la arena de la playa, "signo de la playa"15 (Figura 3b).

En un estudio de 58 pacientes, comparativo, entre los diferentes signos ultrasonográficos para diferenciar la inflamación pulmonar aguda del síndrome de distrés respiratorio agudo (SDRA) y del edema pulmonar agudo cardiogénico, todos los pacientes con SDRA y no así en aquellos con edema pulmonar, tuvieron engrosamiento de los septos interlobares en una distribución homogé- nea y bilateral, en especial en los campos pulmonares anteriores. Además, tuvieron acentuación del "signo de cometa" intercaladas con áreas de pulmón normal en un patrón parcheado, áreas de consolidación del parénquima pulmonar con broncograma aéreo sobre todo en las áreas posteriores. El signo de "deslizamiento pulmonar" estuvo reducido o ausente y, finalmente, la línea pleural apareció irregular y engrosada con consolidaciones subpleurales pequeñas, mientras que en el edema pulmonar la línea pleural se pudo definir claramente. Otro signo que diferencia el SDRA del edema pulmonar es la presencia de líquido pleural, en el primero fue escaso y en el segundo fue significativo. En este estudio, los autores concluyeron que el ultrasonido torácico es un método efectivo para diferenciar estas patologías entre sí $^{16}$.

En otras condiciones, como la neumonía lobar, se puede observar ecogenicidad difusa e irregular, similar al hígado, especialmente en fase inicial de la neumonía. En la contusión pulmonar y en atelectasia lobar se observa pérdida de aireación del parénquima pulmonar. La consolidación alveolar se evidencia por la imagen hipoecoica pobremente definida en forma de cuña. Esto contrasta con la imagen hiperecoica puntiforme dentro de la consolidación, que corresponden a la presencia de gas, $\mathrm{y}$ es el correspondiente al signo de broncograma aéreo (Figura 4). En fases posteriores, tras uso de antibiótico, se puede detallar imágenes aéreas en progreso en el interior de las consolidaciones neumónicas, signos de curación del parénquima pulmonar. La ultrasonografía tiene más sensibilidad que la radiografía convencional en identificar necrosis y abscesos, los cuales se observan como imágenes nodulares con márgenes irregulares o mal definidas, de contenido heterogéneo, con ecos internos o anecogénico ${ }^{3}$.

Con la inspiración hay reforzamiento de es-
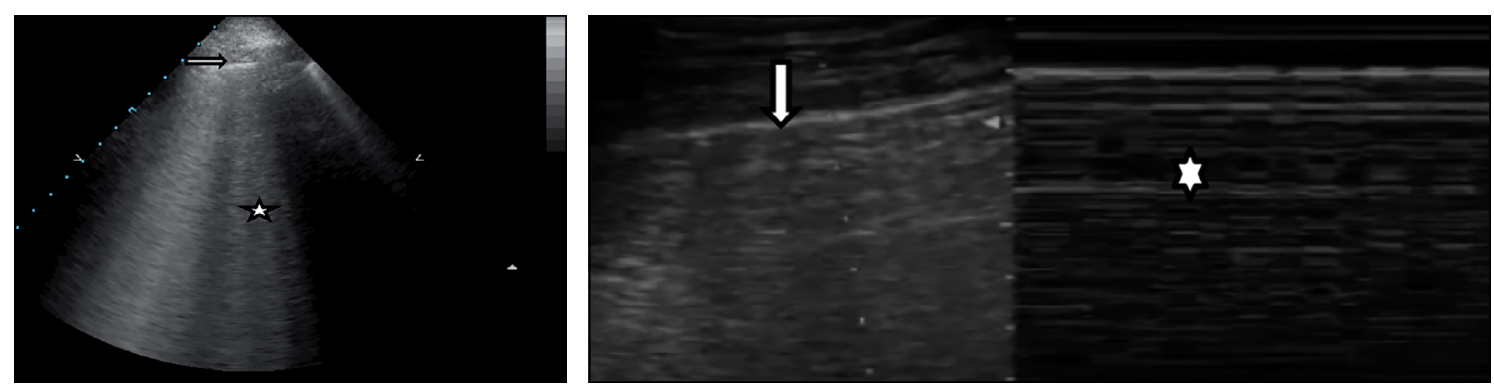

Figura 3. A: Pleura (flecha), cola de cometa (asterisco); B: signo de la playa. 


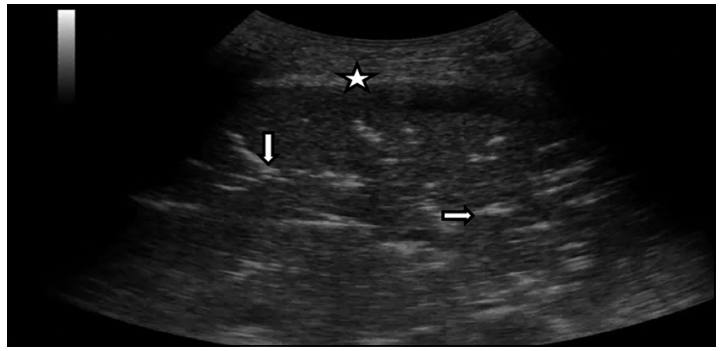

Figura 4. Consolidación e imágenes lineales hiperecogénicas ramificadas (Broncograma-flechas), pleura (asterisco).

tos puntos hiperecoicos debido al aire. Ha sido descrita la utilidad del ultrasonido torácico para el seguimiento de maniobras de reclutamiento alveolar en pacientes en ventilación mecánica, pudiéndose observar cambios en la ecogenicidad del parénquima pulmonar como signo de redistribución del líquido intersticial. Sin embargo, faltan estudios de seguimiento para definir una correlación entre los datos ultrasonográficos y datos clínicos.

\section{Ultrasonido para diagnóstico de neumotórax}

La presencia de aire en la cavidad pleural anula los artefactos que son vistos en el pulmón normal, como el signo del "deslizamiento" (deslizamiento del parénquima pulmonar adyacente a la pleura visceral) y la "cola de cometa" (presencia de aire en los alvéolos). La ausencia de estos signos, más la presencia en Modo-B de la imagen de bandas obscuras (aire) en medio de las líneas hiperecoicas (tejido pulmonar) hacen sospechar el neumotórax. En Modo-M se puede apreciar la imagen de múltiples líneas horizontales paralelas de dife- rentes grados de ecogenicidad a todo lo largo del campo visual en la pantalla, a lo que se le ha dado el nombre de "signo de la estratósfera" (Figura 5).

El diagnóstico de neumotórax por ultrasonido requiere de mayor experiencia por parte del operador, en especial para diferenciar un neumotórax parcial. Se describe, además de la ausencia del signo de "deslizamiento" pulmonar, el signo del "punto pulmonar", en el que hay transición de la imagen granular del parénquima pulmonar a una imagen diferente de líneas horizontales que representa la ausencia de tejido pulmonar. La sensibilidad y especificidad para diagnosticar neumotórax en pacientes en sala de emergencia o UCI fue de $86 \%$ y $97 \%$ respectivamente. En personas entrenadas, el ultrasonido puede dar el diagnóstico de neumotórax en 2 a 4 minutos, contrario a la radiografía de tórax convencional que puede demorar 20 a $30 \mathrm{~min}^{17}$.

Aunque se solía confinar el uso del ultrasonido al radiólogo, esto ha sido cambiado por la necesidad de que los médicos encargados de hacer procedimientos invasivos al pie de la cama del paciente tengan la opción de realizarlos con reducción de riesgos, tanto en el abordaje de estudio en pacientes con patologías no agudas, como también en pacientes en quienes el riesgo de mortalidad es elevado como el neumotórax y el edema pulmonar ${ }^{18,19}$.

\section{Ultrasonido en la traqueostomía percutánea}

La traqueotomía por dilatación percutánea (TDP) es un procedimiento frecuente en unidades de cuidados intensivos. El sangrado durante el procedimiento y a los días posteriores, pudiese corresponder a daño de ramas de la vena yugular

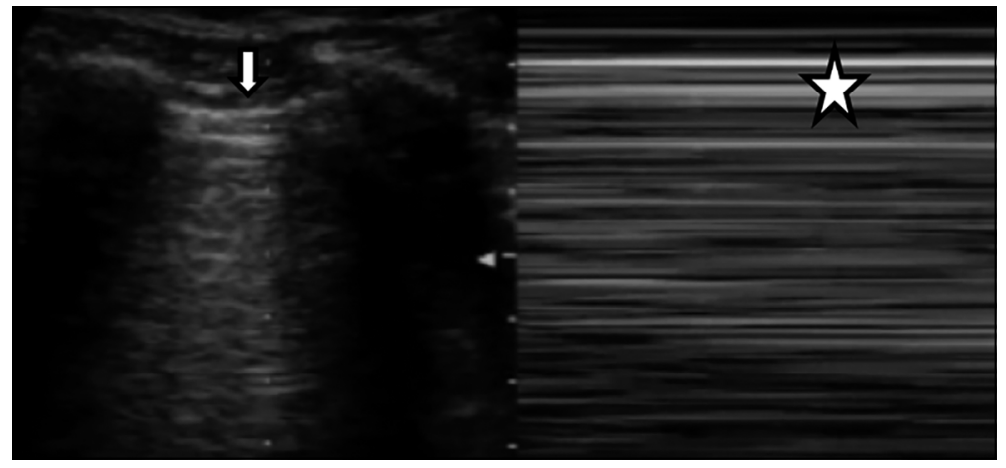

Figura 5. Pleura (flecha), signo de la estratósfera (asterisco). 
externa o tiroidea, como también a un sangrado que implica una alta tasa mortalidad, como el de la arteria innominada. El ultrasonido de la zona supraesternal hasta el hioides facilita la visualización de estructures vasculares y del tejido tiroideo, e identifica el punto más apropiado para la punción traqueal y ostomía. Especial utilidad tiene en paciente con obesidad mórbida, donde es más difícil demarcar estructuras anatómicas y vasculares. Además, la ultrasonografía puede identificar la anatomía aberrante ${ }^{20,21}$.

\section{Conclusiones}

El ultrasonido torácico representa una técnica de apoyo con claras ventajas para tomar decisiones y dirigir procedimientos invasivos. Incrementa la seguridad para el paciente, disminuye costos y tiempo. Hay suficiente evidencia sobre su utilidad en patologías pleurales, mediastínicas y pulmonares, así como procedimientos terapeúticos como la traqueotomía percutánea y la colocación de drenaje pleurales. El ultrasonido está revolucionando la práctica de los neumólogos, siendo una herramienta importante para la Neumología Intervencionista. Los neumólogos que se interesen pueden ser entrenados para hacer acopio de esta técnica y hacerla una parte integral de la práctica diaria clínica.

\section{Referencias}

1. Ihnatsenka B, Boezaart AP. Ultrasound: Basic understanding and learning the language. Int J Shoulder Surg 2010; 4 (3): 55-62.

2. Bouhemad B, Zhang M, Lu Q, Rouby JJ. Clinical review: Bedside lung ultrasound in critical care practice. Crit Care 2007; 11 (1): 205.

3. Vollmer I, Gayete A. [Chest ultrasonography]. Arch Bronconeumol 2010; 46 (1): 27-34.

4. Koegelenberg C, Bollinger C. Textbook of Pleural Diseases. London. Taylor \& Francis. 2008.

5. Ernst A, Feller-Kopman. Ultrasound-Guided Procedures and Investigations. A manual for the Clinician. New York, Taylor \& Francis Goup. 2006.

6. Vignon P, Chastagner C, Berkane V, Chardac E, Francois B, Normand S, et al. Quantitative assessment of pleural effusion in critically ill patients by means of ultrasonography. Crit Care Med 2005; 33 (8): 1757-63.

7. Eibenberger KL, Dock WI, Ammann ME, Dorffner R, Hormann MF, Grabenwoger F. Quantification of pleural effusions: sonography versus radiography. Radiology 1994; 191 (3): 681-4.

8. Currie GP, Alluri R, Christie GL, Legge JS. Pneumothorax: an update. Postgrad Med J 2007; 83 (981): 461-5.

9. Stokes LS. Percutaneous management of malignant fluid collections. Semin Intervent Radiol 2007; 24 (4): 398-408.

10. Sartori S, Tombesi P. Emerging roles for transthoracic ultrasonography in pleuropulmonary pathology. World J Radiol 2010; 2 (2): 83-90.

11. Staes W, Funaki B. "Ex vacuo" pneumothorax. Semin Intervent Radiol 2009; 26 (1): 82-5.

12. Ho TB, Alexander P, Cooke NT. A rapidly increasing pleural effusion. J R Soc Med 2004; 97 (1): 25-6.

13. Lyn-Kew KE, Koenig SJ. Bedside ultrasound for the interventional pulmonologist. Clin Chest Med 2013; 34 (3): 473-85.

14. British Thoracic Society Standards of Care C. BTS statement on malignant mesothelioma in the UK, 2007. Thorax 2007; 62 Suppl 2: ii1-ii19.

15. Gargani L. Lung ultrasound: a new tool for the cardiologist. Cardiovasc Ultrasound 2011; 9: 6.

16. Copetti R, Soldati G, Copetti P. Chest sonography: a useful tool to differentiate acute cardiogenic pulmonary edema from acute respiratory distress syndrome. Cardiovasc Ultrasound 2008; 6: 16.

17. Beckh S, Bölcskei PL, Lessnau KD. Real-time chest ultrasonography: a comprehensive review for the pulmonologist. Chest 2002; 122 (5): 1759-73.

18. Noble VE, Lamhaut L, Capp R, Bosson N, Liteplo A, Marx JS, et al. Evaluation of a thoracic ultrasound training module for the detection of pneumothorax and pulmonary edema by prehospital physician care providers. BMC Med Educ 2009; 9: 3.

19. Hoppmann RA, Rao VV, Poston MB, Howe DB, Hunt PS, Fowler SD, et al. An integrated ultrasound curriculum (iUSC) for medical students: 4-year experience. Crit Ultrasound J 2011; 3 (1): 1-12.

20. Rudas M, Seppelt I. Safety and efficacy of ultrasonography before and during percutaneous dilatational tracheostomy in adult patients: a systematic review. Crit Care Resusc 2012; 14 (4): 297-301.

21. Kundra P, Mishra SK, Ramesh A. Ultrasound of the airway. Indian J Anaesth 2011; 55 (5): 456-62. 